\section{Eyelid Myotonia and Face Stiffness in Skeletal Muscle Sodium Channelopathy}

Srijan Adhikari, MBBS'; Jeffrey Statland, MD';

Constantine Farmakidis, $\mathrm{MD}^{1}$

${ }^{1}$ University of Kansas Medical Center

Keywords: myotonia, paramyotonia congenita, nondystrophic myotonia, eyelid myotonia, skeletal muscle sodium channelopathy, skeletal muscle chloride channelopathy

A 70-year-old man presented with lifelong muscle stiffness and pain. This improved with repetitive muscle activity consistent with a warmup phenomenon and worsened with exposure to cold leading to painful facial cramping, paroxysmal attacks of leg stiffness with falls and throat spasms following a cold drink.

The neurologic examination showed well-developed muscles (Herculean musculature), grip myotonia and prominent delayed relaxation of the eyelids after forced eye closure (eyelid myotonia, video 1). Genetic testing revealed a heterozygous known pathogenic variant (p. Argl448Cys) in the $S C N 4 A$ gene diagnostic of a skeletal muscle sodium channelopathy, a non-dystrophic myotonic disorder.

Myotonia is the clinical phenomenon of delayed skeletal muscle relaxation following voluntary contraction or percussion. It is seen in the dystrophic myotonic disorders (myotonic dystrophy type 1 and 2) which are disorders of progressive weakness and multisystem involvement notable for early cataract formation and potential cardiac involvement. Myotonia is also seen in nondystrophic myotonic disorders, as in this patient. These are characterized by exclusive skeletal muscle involvement with features of delayed muscle relaxation and stiffness, muscle pain, fatigue and sometimes weakness. ${ }^{1}$

Non-dystrophic myotonias are caused by mutations in two skeletal muscle voltage-gated ion channels that lead to muscle membrane hyperexcitability: (1) gain-offunction mutations in the voltage-gated sodium ion channel (SCN4A), and (2) loss-of-function mutations in the chloride ion channel (CLCNI).

In sodium skeletal muscle channelopathies, eyelid myotonia, facial stiffness, and exacerbation of symptoms in cold weather are more common than in chloride channelopathies. ${ }^{1}$ This constellation of symptoms is seen in this patient and illustrates how aspects of the myotonia syndrome and examination finding of eyelid myotonia can suggest $S C N 4 A$ as the more likely causative gene on clinical grounds alone.

Historically, patients with non-dystrophic myotonia exhibiting unexpected or "paradoxical" worsening of myotonia with exertion have been described as having paramyotonia congenita-a subtype of muscle channelopathy later linked specifically to the $S C N 4 A$ gene., ${ }^{2,3}$ The patient reported here also has an $S C N 4 A$ pathogenic sequence change but does not fit the disease description for paramyotonia congenita due to consistently reported attenuation of stiffness with muscle exertion or a warm-up phenomenon. Notably, while the warm-up phenomenon is more common in chloride channelopathy it is not exclusive to chloride channel disease and has been reported in $35 \%$ of subjects with $S C N 4 A$ mutations in a prospective observational study of 34 subjects. ${ }^{4-6}$

Video 1 highlights the patient's personal experience with skeletal muscle sodium channelopathy. Here, the worsening of symptoms with cold exposure was sufficiently severe to prompt the patient to maintain a full beard in an effort to lessen the burden of face stiffness and pain that are most severe in cold weather.

The management of sodium and chloride skeletal muscle channelopathies is symptomatic. Mexiletine, a sodium channel blocking class IB antiarrhythmic, is a firstlineagent for themanagement of myotoniainnon-dystrophic myotonias and has the most evidence of effectiveness. ${ }^{7}$ Other sodium channel blockers, lamotrigine, ranolazine

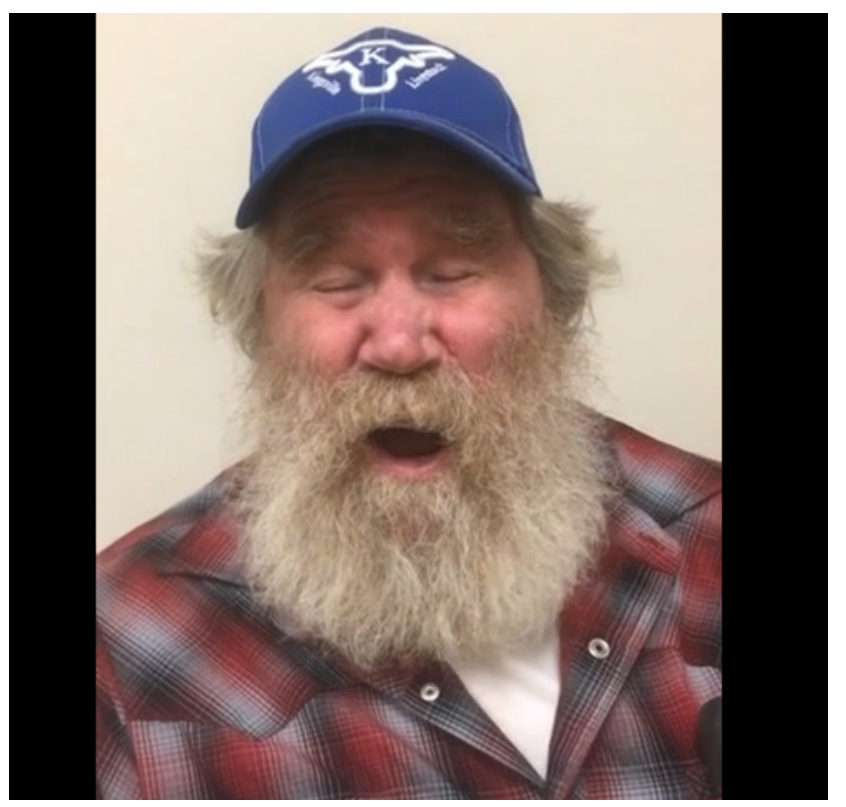

Figure 1. Still from video example of delayed relaxation of the eyelids after forced eye closure (eyelid myotonia). 
and the carbonic anhydrase inhibitor, acetazolamide, have also shown clinical benefit and may also be used.

In addition, this patient should be informed about the potential of severe generalized muscle stiffness or myotonic crisis that can occur with the use of the depolarizing muscle relaxant succinylcholine during general anesthesia. ${ }^{8}$ Having a medical warning card or wrist band that displays the nondystrophic myotonia diagnosis and drug contraindications may further help mitigate perioperative risk.

\section{Video 1. Eyelid Myotonia}

$\underline{\text { Video } 1 .}$

The media clip captures a pronounced example of delayed relaxation of the eyelids after forced eye closure (eyelid myotonia). Also, note the full facial hair that the patient cites as a helpful measure for mitigating facial muscle stiffening and pain in cold weather.

\section{Corresponding Author}

Srijan Adhikari, MBBS,

Department of Neurology,

University of Kansas Medical Center,

3901 Rainbow Boulevard, MS 2012,

Kansas City, KS 66160

sadhikari@kumc.edu

\section{References}

1. Stunnenberg BC, Lorusso S, Arnold WD, et al. Guidelines on clinical presentation and management of nondystrophic myotonias. Muscle \& Nerve. 2020; https:// doi.org/10.1002/mus.26887
2. Ngrams - Paramyotonia Congenita. Google Books. https://books.google.com/ngrams/graph?conte $\underline{\mathrm{nt}=\text { paramyotonia }+ \text { congenita\&year_start }=1800 \& \text { year }}$ $\underline{\text { end }=2019 \& \text { corpus }=26 \& \text { smoothing }=3 \& \text { direct }}=$ url=tl $\% 3 \mathrm{~B} \% 2$ Cparamyotonia $\% 20$ congenita $\% 3 \mathrm{~B} \% 2 \mathrm{CcO}$. 'Accessed' March 292021

3. Ptáček LJ, George AL, Barchi RL, et al. Mutations in an S4 segment of the adult skeletal muscle sodium channel cause paramyotonia congenita. Neuron. 199205-01 1992;8(5); 891-897. https://doi.org/10.1016/0896$\underline{6273(92) 90203-p}$

4. Trivedi JR, Bundy B, Statland J, et al. Nondystrophic myotonia: prospective study of objective and patient reported outcomes. Brain. 2013-07-01 2013;136(7); 2189-2200. https://doi.org/10.1093/brain/awtl33

5. Trip J, Faber CG, Ginjaar HB, Engelen BGM, Drost G. Warm-up phenomenon in myotonia associated with the V445M sodium channel mutation. Journal of Neurology. 2007-02-01 2007;254(2); 257-258. https://doi. org/10.1007/s00415-006-0353-2

6. Lossin C. Nav 1.4 slow-inactivation: Is it a player in the warm-up phenomenon of myotonic disorders? Muscle \& Nerve. 2013-04-01 2013;47(4); 483-487. https://doi. org $/ 10.1002 /$ mus.23713

7. Statland JM. Mexiletine for Symptoms and Signs of Myotonia in Nondystrophic Myotonia. JAMA. 2012;308(13); 1357. $\underline{\mathrm{https}: / / \mathrm{doi} . o r g / 10.100 \mathrm{l} /}$ jama.2012.12607

8. Bandschapp O, Iaizzo PA. Pathophysiologic and anesthetic considerations for patients with myotonia congenita or periodic paralyses. 2013;23(9); 824-833. https://doi.org/10.1111/pan.12217 